\title{
High-Resolution Vessel Wall MR Imaging as an Alternative to Brain Biopsy
}

W e commend Zeiler et $\mathrm{al}^{1}$ for their study entitled "Vessel Wall MRI for Targeting Biopsies of Intracranial Vasculitis," recently published in American Journal of Neuroradiology, in which the authors tested the applicability of contrast-enhanced, high-resolution 3D vessel wall MR imaging for the detection of vascular inflammation and assistance with direct open biopsies of intracranial vessels and adjacent brain parenchyma. The authors concluded that this MR imaging technique could be used to identify inflamed intracranial vessels, enabling precise biopsy localization guidance.

Traditionally, biopsy is considered essential for a definitive diagnosis of central nervous system vasculitis and exclusion of similar-appearing conditions. ${ }^{2}$ Given the invasiveness of biopsy, we believe there should be consideration of whether it may be replaced with high-resolution vessel wall MR imaging, especially in patients with secondary vasculitis. In this context, we present the case of a 37-year-old man with a history of headache, right hemiparesis, and reduced consciousness. He had no skin lesions. A 3T MR imaging study in this patient showed an acute stroke in the left nucleocapsular region and vasogenic edema in the brain parenchyma around the horizontal segment of the left middle cerebral artery. MR angiography showed no changes. High-resolution vessel wall imaging showed smooth and concentric wall thickening and enhancement of the left MCA. White blood cell and protein levels were elevated in the patient's CSF. Polymerase chain reaction for Varicella zoster virus was positive in the CSF. The patient was treated for presumed Varicella zoster virus vasculitis with acyclovir and pulse therapy with corticosteroids. After the treatment, the patient recovered clinically with headache alleviation and an improvement in his level of consciousness. Posttreatment high-resolution vessel wall MR imaging showed a reduction in the arterial wall thickening and enhancement (Figure).

Brain biopsy retains an essential role in the diagnosis of CNS vasculitis and is the criterion standard for the diagnosis of primary CNS vasculitis. Some authors even recommend that a biopsy, including cortical and meningeal tissue, be performed in all sus-

http://dx.doi.org/10.3174/ajnr.A5950 picious cases if possible. ${ }^{2}$ However, these articles were published when high-resolution vessel wall MR imaging was an emergent technique and larger studies examining it had yet to be reported. Since then, vessel wall imaging has become an important tool for evaluating vascular diseases and physicians are becoming increasingly familiar with changes revealed by this technique. Furthermore, an open cranium operation, even for a simple biopsy, has serious risks, including infection, skull fracture, and hemorrhage. Such complications can increase the morbidity and mortality risks, prolong postoperative recovery, and delay treatment. Avoiding an operation eliminates the risk of surgical complications and reduces the cost of care.

Similar to our patient, patient number 6 of Zeiler et $\mathrm{al}^{1}$ had brain vasculitis secondary to Varicella zoster virus. However, we did not perform a biopsy to confirm the diagnosis in our patient. Other authors have also published cases of Varicella zoster brain vasculitis without brain biopsy data or with inconclusive biopsy results in which high-resolution intracranial vessel wall MR imaging was used to diagnose and track the treatment response. ${ }^{3}$ Although Obusez et $\mathrm{al}^{4}$ have seen inconsistent results in follow-up vessel wall imaging findings after treatment of primary CNS vasculitis, the potential role of this technique as a marker of treatment response in CNS vasculitis should continue to be investigated because there is a heterogeneity of disease activity and patient response to treatment. Also, there is a lack of longitudinal studies evaluating the role of this technique in tracking treatment response in secondary CNS vasculitis.

Hence, the growing clinical applicability of intracranial vessel wall imaging has contributed to making this technique part of a state-of-the-art MR imaging protocol for vascular disease diagnosis. Physicians should be aware that vessel wall imaging may be used to avoid invasive procedures such as brain biopsy and to track treatment response.

\section{REFERENCES}

1. Zeiler SR, Qiao Y, Pardo CA, et al. Vessel wall MRI for targeting biopsies of intracranial vasculitis. AJNR Am J Neuroradiol 2018;39: 2034-36 CrossRef Medline

AJNR Am J Neuroradiol 40:E17-E18 Mar 2019 www.ajnr.org E17 
2. Dutra LA, de Souza AW, Grinberg-Dias G, et al. Central nervous system vasculitis in adults: an update. Autoimmun Rev 2017;16:123-31 CrossRef Medline

3. Mossa-Basha M, Alexander M, Gaddikeri S, et al. Vessel wall imaging for intracranial vascular disease evaluation. J Neurointerv Surg 2016; 8:1154-59 CrossRef Medline

4. Obusez EC, Hui F, Hajj-Ali RA, et al. High-resolution MRI vessel wall imaging: spatial and temporal patterns of reversible cerebral vasoconstriction syndrome and central nervous system vasculitis. AJNR Am J Neuroradiol 2014;35:1527-32 CrossRef Medline
D.G.G. Corrêa

Clínica de Diagnóstico por Imagem Rio de Janeiro, Brazil

Department of Radiology

Hospital Universitário Clementino Fraga Filho Federal University of Rio de Janeiro

Rio de Janeiro, Brazil

(1) L.C. Hygino da Cruz Jr.

Clínica de Diagnóstico por Imagem Rio de Janeiro, Brazil
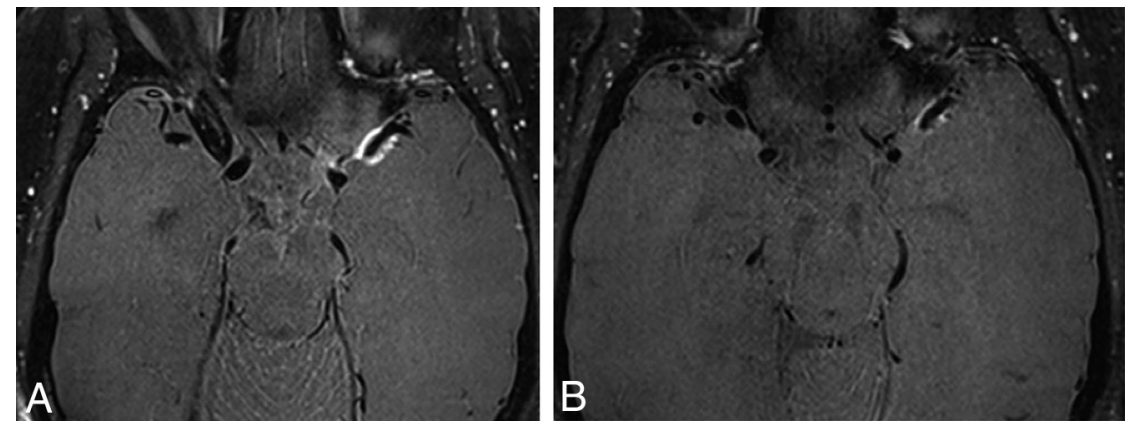

FIGURE. Brain vasculitis secondary to Varicella zoster virus infection. A, Intracranial vessel wall imaging after intravenous contrast injection revealing smooth, concentric arterial wall thickening and enhancement of the horizontal segment of the left MCA, compatible with inflammatory vasculopathy. $B$, Posttreatment intracranial vessel wall imaging shows diminished vessel wall thickening and enhancement. 